\title{
FULL TERM EXTRAUTERINE ABDOMINAL PREGNANCY
}

Neetu Ahirwar ${ }^{1}$, Padma Shukla², Pappy Natung ${ }^{3}$

HOW TO CITE THIS ARTICLE:

Neetu Ahirwar, Padma Shukla, Pappy Natung. "Full Term Extrauterine Abdominal Pregnancy". Journal of Evolution of Medical and Dental Sciences 2014; Vol. 3, Issue 08, February 24; Page: 1895-1898,

DOI: $10.14260 /$ jemds/2014/2075

ABSTRACT: Extrauterine abdominal pregnancy is extremely rare and is frequently missed during antenatal care, despite the routine use of obstetrics ultrasound. A 23 year old primigravida at 39 weeks of gestation was admitted to the department on December 2, 2012, in routine hour with transverse lie with oligohydramnios. On examination her vitals and laboratory investigation were within normal limits. One of the ultrasonographic scanning done by radiologist at $35 \mathrm{wk}$. 3 days gestation demonstrated a single live intrauterine fetus of CGA 33 wk. 1 day with transverse lie, fundal gr II placenta and AFI- $10 \mathrm{~cm}$. Ultrasonography done at the department on the day of admission demonstrated single live intrauterine fetus with transverse lie and AFI-3.8 cm and EFW 2.4 Kg and placenta was right anterior.. She was planned for elective caesarean section on account of transverse lie and oligohydramnios at term. At laparotomy the following findings were made: Secondary abdominal pregnancy arising from right tubal ectopic rupture and making pseudogestational sac with meconium stained liquor. Omentum, right tube and ovary were adhered to sac. The uterus and left tube and ovary were healthy and normal. Other abdominal organs were normal. A $1.5 \mathrm{Kg}$ live male baby with good Apgar score and incompletely formed mandible extracted from pseudogestational sac. There was significant bleeding from pseudogestational sac and patient went in shock during intraoperative period. One unit whole blood transfused during intraoperative period. Placenta was adherent to the pseudogestational sac so Psudogestational sac was removed with placenta in situ and right sided salpingo-oophrectomy was also done due to their adherence. Hemostasis was secured. The patient and baby progressed well and were discharged on the seventh postoperative day.

KEYWORDS: abdominal pregnancy, live baby, term pregnancy.

INTRODUCTION: Ectopic pregnancy represents about 1-2\% of all pregnancies with 95\% occurring in the fallopian tube. Abdominal pregnancies represent just about $1 \%$ of ectopic pregnancies ${ }^{1}$. The incidence of abdominal pregnancy ranges between 1: 10000 pregnancies to 1:30,000 pregnancies. ${ }^{1,2}$

Abdominal pregnancy with live birth is rare. The diagnosis of abdominal pregnancy is frequently missed during antenatal care, despite the routine use of abdominal ultrasound. However, it is important to detect an extrauterine abdominal pregnancy because the associated maternal mortality is 90 times greater than with an intrauterine pregnancy and 8 times greater than the tubal pregnancy ${ }^{3}$. Survival of the newborn is also affected with perinatal mortality rate of $40 \%$ to $95 \% .{ }^{4}$

This is a report of full term abdominal pregnancy in primigravida with live fetus. Laparotomy was performed. Both mother and baby were discharged in healthy condition.

CASE REPORT: A 23 year old primigravida at 39 weeks of gestation was admitted to the department on December 2, 2012, in routine hours with transverse lie with oligohydramnios. On examination her vitals and laboratory investigation were within normal limit. The abdominal examination revealed fundal height 32 wk., transverse lie with FHR $130+\mathrm{R}$ and no uterine contraction. First ultrasonographic scanning was done by a radiologist at 35 week gestation and demonstrated a single 
live intrauterine fetus of CGA 33 week 1 day with transverse lie and fundal gr II placenta and AFI$10 \mathrm{~cm}$. Ultrasonography was done in the department on the day of admission demonstrated single live intrauterine fetus with transverse lie and AFI-3.8 with EFW 2.4 and placenta rt anterior. She was planned for elective caesarean section on account of transverse lie and oligohydramnios at term, in routine hours after arrangement of blood under spinal anesthesia.

On opening the abdomen with pfannensteil incision, omentum was found to be adherent to the pregnancy sac which was outside the empty uterus. On opening the pseudogestational sac, liquor was found meconium stained and a $1.5 \mathrm{~kg}$ live baby was extracted with a good APGAR score and incompletely formed mandible. On further exploration secondary abdominal pregnancy was diagnosed originating from ruptured right tubal ectopic pregnancy and right ovary was adherent to the sac. The uterus and left tube and ovary were healthy and normal. Other abdominal organs were normal. There was significant bleeding from pseudogestational sac and patient went in shock .One unit whole blood transfused during intra op period. Placenta was adherent to the pseudosac so Psudosac was removed with placenta in situ and right sided salpingo-oophrectomy was also done due to their adherence. Hemostasis was secured. Postoperative period was uneventful. The mother and baby progressed well and were discharged on the seventh postoperative day.

DISCUSSION: Extrauterine abdominal pregnancy beyond 20 weeks gestation with a viable fetus is a rare condition, with an estimated prevalence of one out of 8099 hospital deliveries. It is classified into two types. Primary abdominal pregnancy where implantation of the fertilized ovum occurs directly in the abdominal cavity with the fallopian tubes and ovaries being intact. In contrast, secondary abdominal pregnancy occurs following an extra uterine pregnancy that ruptures and gets reimplanted within the abdomen with the evidence of tubal or ovarian damage.

Diagnosis of abdominal pregnancy continues to be a challenge, resulting in delay in diagnosis and decision making. Even in ideal conditions a sonographic diagnosis of abdominal pregnancy is missed in half of the cases.5, 6

It is interesting to note that patients with an extra uterine abdominal pregnancy typically have persistent abdominal pain and or gastrointestinal symptom during pregnancy. ${ }^{7}$ This patient, however, did not have any symptom during her pregnancy.

Ramachandran et al (2004) reported a case of undiagnosed abdominal pregnancy, only diagnosed at the time of cesarean section carried out for a persistent oblique lie. Delivery of the fetus was followed by near catastrophic hemorrhage. The portion of the placenta was non-resectable and following surgery patient was sent to intensive care unit 7 . In the present patient sonography, reporting was, Intrauterine pregnancy with oligohydramnios with persistent transverse lie and as usual abdominal pregnancy was diagnosed at the time of cesarean section and patient went in shock during intraoperative period.

About $21 \%$ of babies born after an extrauterine abdominal pregnancy have birth defects, presumably due to compression of the fetus in the absence of the amniotic fluid buffer. Typical deformities include limb defects, facial and cranial asymmetry, joint abnormalities and central nervous system malformation. ${ }^{8}$ In this case baby was having incompletely formed mandible along with severe oligohydramnios. 
Magnetic resonance imaging and serum alfa fetoprotein have been used to diagnose abdominal pregnancy 9 ,10, however there was no justification to perform these tests in this patient as the diagnosis was not suspected.

CONCLUSION: This is a report of an extrauterine abdominal pregnancy which was most likely implanted in right fallopian tube and ruptured, later on developed as secondary abdominal pregnancy. The pregnancy continued uneventfully to full term and ended successfully with operative delivery of live baby with incompletely formed mandible. The importance of this report is the fact that an extrauterine abdominal pregnancy could be missed during antenatal period despite ultrasound examinations. Bleeding is the single most important life-threatening complication for the mother while fetal malformation is one of the numerous challenges that can confront the newborn. Furthermore, the antenatal diagnosis of advanced extrauterine pregnancy does not necessarily justify the termination of the pregnancy since good maternal and fetal outcome is not uncommon.

\section{REFERENCES:}

1. Nwobodo EI. Abdominal pregnancy. A case report. Ann Afr Med. 2004; 3(4):195-196.

2. Badria L, Amarin Z, Jaradat A, Zahawi H, Gharaibeh A. Full-term viable abdominal pregnancy. A case report and review. Arch Gynaecol Obstet. 2003; 268(4):340-342.

3. Sapuri M, Klufio C. A case of advanced viable extra uterine pregnancy .P NG Med J .1997 Mar, 40 (1): 44-47.

4. Martin JN Jr, Sessums JK, Martin RW, Pryor JA, Morrison JC. Abdominal pregnancy: current conceptus of management. Obstet Gynecol. 1988 Apr;71(4):549-57.

5. Cotter AM, Jacques EG, Izquierdo LA. Extended field of view sonography: a useful tool in the diagnosis and management of abdominal pregnancy. J Clin Ultrsound 2004 May; 32(4):207210.

6. Costa SD, Presley J, Bastert G. Advanced abdominal pregnancy. Obstet Gynecology Surv, 1991 Aug; 46(8):515-525.

7. Ramachandran K, Kirk P. Massive hemorrhage in a previously undiagnosed abdominal pregnancy presenting for elective cesarean delivery .Can J Anaesth.2004 Jan; 51(1):57-61.

8. Stevens CA. Malformations and deformations in abdominal pregnancy. Am J Med Genet 1993, 47:1189-1195. PubMed Abstract | Publisher Full Text

9. Krishna D, Damyanti S. Advanced abdominal pregnancy: a diagnostic and management dilemma. J Gynecol Surg 2007, 23:69-72.

10. Tromans PM, Coulson R, Lobb Mo,Abdulla U. Abdominal pregnancy associated with extremely elevated serum alfa-protein: case report. Br J Obstet Gynaecol. 1984 Mar;91(3):296-8. 


\section{AUTHORS:}

1. Neetu Ahirwar

2. Padma Shukla

3. Pappy Natung

\section{PARTICULARS OF CONTRIBUTORS:}

1. Assistant Professor, Department of Obstetrics and Gynaecology, S. S. Medical College and Associated S. G. M. Hospital, Huzur Rewa, (M. P.)

2. Assistant Professor, Department of Obstetrics and Gynaecology, S. S. Medical College and Associated S. G. M. Hospital, Huzur Rewa, (M. P.)
3. Post Graduate Student, Department of Obstetrics and Gynaecology, S. S. Medical College and Associated S. G. M. Hospital, Huzur Rewa, (M. P.)

\section{NAME ADDRESS EMAIL ID OF THE CORRESPONDING AUTHOR:}

Dr. Neetu Ahirwar, F-19, Doctor's Colony, Medical College Campus, Huzur, Rewa, (M. P.)

E-mail: neetuahirwarbharang@gmail.com

Date of Submission: 27/01/2014. Date of Peer Review: 28/01/2014. Date of Acceptance: 06/02/2014. Date of Publishing: 19/02/2014. 